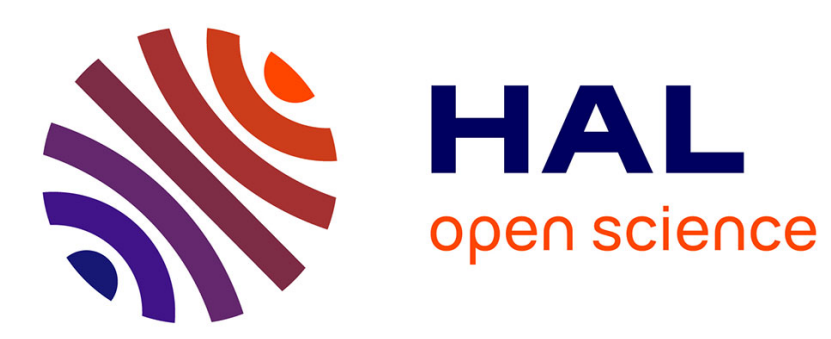

\title{
Total Domination of Graphs and Small Transversals of Hypergraphs
}

\author{
Stéphan Thomassé, Anders Yeo
}

\section{To cite this version:}

Stéphan Thomassé, Anders Yeo. Total Domination of Graphs and Small Transversals of Hypergraphs. Combinatorica, 2007, 27, pp.473-487. 10.1007/s00493-007-2020-3 . lirmm-00250084

\section{HAL Id: lirmm-00250084 https://hal-lirmm.ccsd.cnrs.fr/lirmm-00250084}

Submitted on 9 Feb 2008

HAL is a multi-disciplinary open access archive for the deposit and dissemination of scientific research documents, whether they are published or not. The documents may come from teaching and research institutions in France or abroad, or from public or private research centers.
L'archive ouverte pluridisciplinaire HAL, est destinée au dépôt et à la diffusion de documents scientifiques de niveau recherche, publiés ou non, émanant des établissements d'enseignement et de recherche français ou étrangers, des laboratoires publics ou privés. 


\title{
Total domination of graphs and small transversals of hypergraphs.
}

\author{
Stéphan Thomassé* \\ and \\ Anders $\mathrm{Yeo}^{\dagger}$
}

\begin{abstract}
The main result of this paper is that every 4-uniform hypergraph on $n$ vertices and $m$ edges has a transversal with no more than $(5 n+4 m) / 21$ vertices. In the particular case $n=m$, the transversal has at most $3 n / 7$ vertices, and this bound is sharp in the complement of the Fano plane. Chvátal and McDiarmid [5] proved that every 3-uniform hypergraph with $n$ vertices and edges has a transversal of size $n / 2$. Two direct corollaries of these results are that every graph with minimal degree at least 3 has total domination number at most $n / 2$ and every graph with minimal degree at least 4 has total domination number at most $3 n / 7$. These two bounds are sharp.
\end{abstract}

\section{Introduction.}

Given a graph $G=(V, E)$, a total dominating set is a subset $S$ of the vertices of $G$ such that every vertex of $G$ has a neighbour in $S$. The minimum size of a total dominating set is the total domination number of $G$. It was proved by Favaron et al. [6] that a graph with $n$ vertices and minimum degree at least 3 has total domination number at most $7 n / 13$. This result has been recently extended to $n / 2$ [4], [2], and a fractional approach can be found in [7]. A transversal in a hypergraph is a subset of vertices which intersects every edge. We are mainly concerned here with transversals of $k$-uniform hypergraphs with the same number of edges and vertices. Precisely, we raise the problem to find the minimum $c_{k}$ for which every $k$-uniform hypergraph with $n$ vertices and $n$ edges has a transversal of size $c_{k} n$. It directly follows that every graph $G$ with minimum degree $k$ and $n$ vertices has a total dominating set with $c_{k} n$ vertices, since in the hypergraph whose edges are the neighbourhoods of the vertices of $G$, a transversal is a total dominating set. The main advantage of considering hypergraphs instead of graphs is that the structure is easier to handle - for instance we can limit ourselves to $k$-uniform structures. When $H$ is a hypergraph on the vertex set $V$ and $X \subseteq V$, we denote by $H \backslash X$ the induced subhypergraph on $V \backslash X$ - that is, we delete all the vertices of $X$, and all the edges having a vertex in $X$.

To fix the ideas, and a bound, let us calculate $c_{2}$ :

Lemma 1 Every 2-uniform hypergraph $H$ has a transversal $T$ such that $3|T| \leq n+m$

Proof. By induction on the number of vertices. If some vertex $x$ has degree at least 2 , we put it in the transversal, and apply the induction hypothesis to $H \backslash x$. Otherwise no vertex has degree 2 and then the edges form a matching, and the formula holds.

\footnotetext{
${ }^{*}$ LIRMM, 161 rue Ada, 34392 Montpellier Cedex 5, France, thomasse@lirmm.fr

${ }^{\dagger}$ Department of Computer Science, Royal Holloway, University of London, Egham; Surrey TW20 0EX, UK (email: yeo@dcs.rhbnc.ac.uk)
} 
Corollary $1 \quad c_{2}=2 / 3$

When $n=3 k$, the extremal graph for $c_{2}$ is certainly a disjoint union of triangles, and this is the sole example by the unicity of the extremal Turán graphs. This kind of example generalizes for all $k$ : If one considers a disjoint union of complete $k$-uniform hypergraphs on $k+1$ vertices, this clearly forms a $k$-uniform hypergraph with $n$ edges and $n$ vertices and the minimum transversal has exactly $2 n /(k+1)$ vertices. By this, we directly have that $c_{k} \geq 2 /(k+1)$. The interesting fact is that equality holds also for $k=3$, that is $c_{3}=1 / 2$, but many extremal examples arise: consider for instance the hypergraph on vertex set $\left\{1, \ldots, n, 1^{\prime}, \ldots, n^{\prime}\right\}$ and edges $\left\{i, i^{\prime}, i+1\right\}$ and $\left\{i, i^{\prime},(i+1)^{\prime}\right\}$ where $i+1$ is understood modulo $n$. However, it turns out that $c_{4}$ is not equal to $2 / 5$, as we will observe. But let us now prove that $c_{3}=1 / 2$. The following result can be found in [5], but we will give an alternative proof as an introduction to the proof of our main result.

Theorem 1 Let $H=(V, E)$ be a 3-uniform hypergraph. There exists a transversal $T$ of $H$ such that $4|T| \leq|V|+|E|$.

Proof. Induction on the number of vertices. If some vertex $v$ has degree at least 3 , we find, by the induction hypothesis, a transversal $T^{\prime}$ of $H \backslash v$. In turn, $T^{\prime} \cup\{v\}$ satisfies our condition. We can now suppose that every vertex has degree at most 2. If some edge $a b c$ satisfies that the degree of $a$ is 2 and the degree of $b$ is 1 , we apply the induction hypothesis to $H \backslash\{a, b\}$ in order to find a transversal $T^{\prime}$ : the transversal $T:=T^{\prime} \cup\{a\}$ still satisfies the hypothesis. If one edge $a b c$ has no vertex of degree 2 , we simply apply the induction hypothesis to $H \backslash\{a, b, c\}$. Now the hypergraph is 2-regular. If the same edge $a b c$ appears twice in $H$, we simply apply the induction hypothesis to $H \backslash\{a, b, c\}$. If not, we can find the following subset of edges

$$
x_{1} y_{1} x_{2}, x_{2} y_{2} x_{3}, x_{3} y_{3} x_{4}, \ldots, x_{k} y_{k} x_{1}
$$

where all the vertices $x_{1}, \ldots, x_{k}, y_{1}, \ldots, y_{k}$ are distinct, because of the following: If two edges intersect on two vertices, then these two edges are of the form $x_{1} y_{1} x_{2}, x_{2} y_{2} x_{1}$. If no two edges intersect on two points, the above sequence is simply obtained by considering a minimum cyclic sequence of edges where only adjacent edges intersect. Now, two cases can happen: If $k=2 l$, choose a transversal $T^{\prime}$ of $H^{\prime}:=H \backslash\left\{x_{i}: i=1 . . k\right\}$ which satisfies the induction hypothesis: the transversal $T:=T^{\prime} \cup\left\{x_{2 i}: i=1 . . l\right\}$ satisfies the hypothesis for $H$ since we deleted $2 l$ vertices and $2 l$ edges to get $H^{\prime}$ from $H$. If $k=2 l+1$, choose a transversal $T^{\prime}$ of $H^{\prime}:=H \backslash\left(\left\{y_{k}\right\} \cup\left\{x_{i}: i=1 . . k\right\}\right)$ which satisfies the induction hypothesis: the transversal $T:=T^{\prime} \cup\left\{x_{2 i}: i=1 . . l\right\} \cup\left\{y_{k}\right\}$ satisfies the hypothesis for $H$ since we deleted $2 l+2$ vertices and $2 l+2$ edges to get $H^{\prime}$ from $H$.

Corollary 2 Every 3-uniform hypergraph $H=(V, E)$ with $|V|=|E|=n$ has a transversal with at most $n / 2$ vertices. In other words, $c_{3}=1 / 2$.

Finally we would like to mention a result of Seymour [9]: every minimal non bipartite hypergraph has at least as many edges as vertices. From this result follows a short proof of the existence of a $n / 2$ transversal in a connected 3-uniform 3-regular hypergraph $H$ with $2 l$ vertices: remove an edge $e$ of $H$ for which $H-e$ is connected ( $e$ exists since there exists a connected spanning subhypergraph with at most $n-2$ edges). Now every proper induced subhypergraph of $H-e$ has less edges than vertices, in particular, by Seymour's result, $H-e$ is two colourable. If $e$ belongs to both colours, we are done. If the color class of $e$ has at most $n / 2$ vertices, it is our transversal. Finally, if it has more than $l+1$ vertices, take the other color class and a vertex of $e$ as a transversal. 


\section{The 4-uniform hypergraphs.}

The case of 4-uniform hypergraphs is much more complicated since what seems to be the extremal case is not the complete 4-uniform hypergraph on 5 vertices but the complement of the Fano plane. Precisely, we consider the hypergraph $\bar{F}$ on the vertex set $\{1,2,3,4,5,6,7\}$ and edge set $\{Q+i: i:=1 . .7\}$, where + is understood modulo 7 and $Q:=\{0,1,2,4\}$ is the set of quadratic residues. In this case we have 7 vertices and we need 3 vertices to hit all the edges. This example provides a graph with 14 vertices, minimum degree 4 and total domination number 6 , simply by considering the incidence bipartite graph of $\bar{F}$. The main result of this section is to prove that these two examples are extremal, in other words we have the following result:

Theorem $2 \quad c_{4}=3 / 7$

We prove a more general formula for 4-uniform hypergraphs which directly implies the value of $c_{4}$. An analog formula was proved in [3], where they established that every 4-uniform hypergraph has a transversal with no more than $(2 n+2 m-d) / 9$ vertices, where $d$ is the number of edges which contain a vertex of degree one. This gives the upper bound $c_{4} \leq 4 / 9$.

An edge of a hypergraph is overlapping if it intersects another edge on at least 2 vertices. An edge is special if it is overlapping and has exactly one vertex of degree 1 . For $k>0$, an edge is $k$-degenerated if it is not special and has exactly $k$ vertices of degree 1 . An edge which is not special and not $k$-degenerated is plain.

Theorem 3 Let $H$ be a 4-uniform hypergraph with $n$ vertices, $p$ plain edges, $s$ special edges and $d$ degenerated edges. There exists a transversal $T$ such that $21|T| \leq 5 n+4 p+3 s / 2+d$.

Proof. Let us consider a counterexample $H$, minimum with respect to its degree sequence, ordered lexicographically (that is, for each hypergraph we order its degree sequence in the decreasing order and we compare two hypergraphs using the lexicographical order on their respective sequences.)

Claim 1 The maximum degree in $H$ is at most three.

Proof. Assume not and remove a vertex $v$ of degree at least 4 . If all its incident edges are plain, the removal of these edges gives at least -16 and the removal of $v$ gives -5 . In all, we get at least -21 , and adding $v$ in the transversal $T^{\prime}$ of $H \backslash v$, the total count is $+21-21$, so this contradicts the minimality of $H$. Now, if some edges incident with $v$ contain degree one vertices, we delete these vertices as well, in this case, the count for removing one edge is at least -6 (that is at least -1 for the removed edge and -5 for the isolated vertex). Again removing all the edges incident with $v$ (plus possibly the now isolated vertices) gives again at least -16 , so we conclude as previously.

In the following of the proof, we will indicate the counting argument we did in the proof of Claim 1 as a sum $+x-y-z-t$ where $x$ is the value of the vertices in the transversal, $y$ is the value of the removed edges, $z$ is the value of the removed vertices, and $t$ is the value change of the edges (since some of the remaining edges can become special or degenerated.)

Claim 2 Every degree two vertex $x$ of $H$ is in a non plain edge.

Proof. If $x$ is in two plain edges $e_{1}, e_{2}$, we split the vertex $x$ into two vertices $x_{1}, x_{2}$, letting $x_{1} \in e_{1}$ and $x_{2} \in e_{2}$. There is a +5 for the new vertex, but at least 2.(-2,5) since $e_{1}, e_{2}$ become at least special. Observe that every transversal of this new hypergraph is also a transversal of $H$, contradicting the minimality of the degree sequence of $H$.

Claim 3 Every vertex $x$ of degree three is incident to at least two plain edges. 
Proof. If not, removing $x$ gives at least $+21-6-15$.

Claim 4 There is no 4-degenerated edge. Sum +21-1-20.

Claim 5 There is no 3-degenerated edge. Sum +21-5-20, in all -4.

Claim 6 Two edges of $H$ do not intersect on 3 vertices.

Proof. Let $e$ and $f$ be two edges intersecting on $x, y, z$. If $e$ is not plain (its degree one vertex being $t$ ), we simply remove the edge $f$ and find a transversal $T^{\prime}$. If $T^{\prime}$ does not contain $t$, it is a transversal of $H$. If it contains $t$, we replace $t$ by any of $x, y, z$. So both are plain. We add then a new vertex $\omega$ to $H$, remove the edges $e, f$ and add the edge $x y z \omega$. Observe that this new graph is lexicographically smaller than $H$, and that the count is $-6,5+5$ in the worst case. Let $T^{\prime}$ be a transversal of this new graph. If $T^{\prime}$ does not contain $\omega$, it is a transversal of $H$. If it contains $\omega$, we replace $\omega$ by any of $x, y, z$ to get a transversal of $H$.

Claim 7 There is no 2-degenerated edge. Sum -2.

Proof. Let $e$ be a 2-degenerated edge. If some vertex of $e$ has degree three, removing it gives at least $+21-9-15$, that is -3 . So there are exactly two vertices $x, y$ of degree two, incident with two edges $e_{x}, e_{y}$, and two vertices of degree one. If $e_{x}=e_{y}$, removing $x$ gives at least $+21-5-20$, so -4. By Claim $6, e_{x}$ and $e_{y}$ do not intersect on three vertices. If $e_{x}$ and $e_{y}$ intersect on two vertices, we remove all the vertices of $e$, we delete $e_{x}$ and $e_{y}$, and add the new edge $\left(e_{x} \cup e_{y}\right) \backslash\{x, y\}$. This new graph has a lexicographically smaller degree sequence than $H$, and any transversal $T^{\prime}$ can be extended by either $x$ or $y$ to form a transversal of $H$. This gives us 21-5-20 if $e_{x}$ and $e_{y}$ are plain. If one is special and the other plain, the sum is 21-5-20. If both $e_{x}$ and $e_{y}$ are special, 21-3-20. Observe that this exhausts all possibilities. Now $e_{x}$ and $e_{y}$ intersect on at most one vertex. If $e_{x}$ and $e_{y}$ are both plain, we remove all the vertices of $e$, we delete $e_{x}$ and $e_{y}$, and add a new edge containing any four vertices of $\left(e_{x} \cup e_{y}\right) \backslash\{x, y\}$. As previously, this gives 21-5-20. If they are not both plain, one vertex $z$ of, say, $e_{x}$ has degree 1 . In this case, we remove all the vertices of $e$, we delete $z$, we delete $e_{x}$ and $e_{y}$, and add a new edge containing any four vertices of $\left(e_{x} \cup e_{y}\right) \backslash\{x, y, z\}$. This gives $21+1-25$.

We will widely use Claim 7 in our proofs. As soon as removing a vertex yields a 2-degenerated edge, we will substract 2 to our sum.

We define the intersection graph of $H$ as the multigraph $G$ on vertex set $V$ and edge set $E$ in the following way: for all pairs $e, f$ of edges of $H$ such that $e \cap f=\{x, y\}$, we add the edge $x y$ to $G$.

Claim 8 The graph $G$ does not have 2-cycles.

Proof. If there are three edges $e, f, g$ pairwise intersecting on $x, y$, removing $x$ gives at least $+21-12-10$. This calculation holds when all the edges are plain. If they are not, we get a better bound.

Claim 9 The graph $G$ has maximum degree 2 .

Proof. Suppose we have three edges $e, f, g$ such that $e \cap f=\{y, x\}, f \cap g=\{y, z\}$ and $e \cap g=\{y, t\}$. If one of $x, z, t$ has degree two, removing $y$ gives at least 21-12-10. Thus, $x, z, t$ have degree 3 , and we let $e_{x}, e_{z}, e_{t}$ their incident other edges. If $e$ is special, by Claim $3, e_{x}$ is plain, and removing $y$ gives 21-9,5-10-2,5. So $e, f, g$ are plain. If $e_{z}$ is non plain, deleting $z$ gives 21-9-10-2,5. Thus all are plain, and we delete $y$ : If $e_{x}=e_{y}=e_{z}$, we have 21-12-5 and an additional -4, since $e_{x}$ becomes 3-degenerated. If $e_{x} \neq e_{y}$, we have 21-12-5 and -5 , since $e_{x}$ and $e_{y}$ become at least special. 


\section{Claim 10 The graph $G$ has maximum degree 1.}

Proof. Suppose we have three edges $e, f, g$ such that $e \cap f=\{x, y\}$ and $f \cap g=\{y, z\}$. By Claim 9, we have $e \cap g=\{y\}$. Clearly $x$ and $z$ have degree 3, otherwise we obtain 21-12-10. We denote respectively by $e_{x}$ and $e_{z}$ the other edges incident to $x$ and $z$. If $g$ is not plain, $e_{z}$ is plain, and thus we can remove $y$, the sum is 21-9-10-2,5. Thus $e, f, g$ are plain. If $e_{z}$ is not plain, we remove $z$ to get 21-9-10-2,5. Thus all edges are plain. If $e_{z} \neq e_{x}$ we remove $y$ to get 21-12-5-5. If $e_{z}=e_{x}$, removing $y$ makes $e_{x}$ at least 2-degenerated. If it is not 4-degenerated, we get 21-12-5-3, and at least an additional -2 by Claim 7 or Claim 5. So the only case left is when removing $y$ makes $e_{x}$ 4-degenerated. Observe that removing $z$ makes $e$ at least 2-degenerated, and again by counting we achieve our contradiction if it is less than 4-degenerated. But this would mean that $e_{x}$ intersects $e$ on at least three vertices, against Claim 6 .

Claim 11 If $x y$ is an edge of $G$, both $x$ and $y$ have degree 3 and are contained in plain edges.

Proof. We consider two edges $e, f$ intersecting on $x, y$. If $x$ has degree two and $y$ has degree three, removing $y$ gives -1 . If they both have degree two, by Claim $2, e$ or $f$ is non plain. We assume that $e$ is non plain and thus special. Observe that $f$ must be plain, otherwise we remove $x$ which gives 21-3-20. Let $z$ be the vertex of $e \backslash f$ with degree at least 2. If $z$ has degree 3, removing $z$ gives at least 21-9,5-10-3, since $f$ becomes at least 2-degenerated. So $z$ has degree two. If the other edge $e_{z}$ of $z$ is plain or special, we are done by removing $x$ : the sum is $21-5,5-15-0,5$. So $e_{z}$ is 1-degenerated. In particular removing $z$ makes $f$ at least 2-degenerated, but not 4-degenerated (since $\left|e_{z} \cap f\right|<2$ ), and in this case the sum is 21-2,5-15-3 and an additional -2 by Claim 7 . So both $x$ and $y$ have degree three, with additional (distinct) edges $e_{x}$ and $e_{y}$. If $e$ is special, by Claim $3, f, e_{x}$ and $e_{y}$ are plain, and we remove $x$ to obtain 21-9,5-10-2,5. So both $e$ and $f$ are plain. If $e_{x}$ is plain and not $e_{y}$, removing $y$ gives 21-9-10-2,5. Suppose that $e_{x}$ and $e_{y}$ are special, removing $x$ gives 21-9,5-10-0,5 and $e_{y}$ is now at least 2-degenerated. The only case to check is when it becomes 4-degenerated. This means that $e_{x}$ and $e_{y}$ intersect on two outside vertices $u v$ with degree 2 , which is impossible since $u v$ would be an edge of $G$, and thus $u, v$ have degree 3. If $e_{x}$ is special and $e_{y}$ is 1-degenerated, removing $y$ gives 21-9-10-0,5, and $e_{x}$ is now 2-degenerated, in all -0,5. So both $e_{x}$ and $e_{y}$ are 1-degenerated, removing $x$ makes $e_{y}$ 2-degenerated, so we have sum 21-9-10 and an additional -2 by Claim 7 .

In particular, we suppose from now on that there are no special edges. When $e, f$ intersect on two vertices $x, y$, and $e_{x}$ is the other edge incident to $x$, we say that $e_{x}$ is an external edge of $\{e, f\}$.

Claim 12 If $g$ is an external edge of $\{e, f\}$ and $g$ is an external edge of $\left\{e^{\prime}, f^{\prime}\right\}$, we have $\{e, f\}=\left\{e^{\prime}, f^{\prime}\right\}$.

Proof. Suppose that $e, f$ intersect on two vertices $x, y$, with external edges $e_{x}, e_{y}$ and $e^{\prime}, f^{\prime}$ intersect on two vertices $x^{\prime}, y^{\prime}$, with external edges $e_{x}^{\prime}, e_{y}^{\prime}$, with the additional condition that $e_{x}^{\prime}=e_{x}$. Here $e_{x}$ plays the role of $g$. Let us prove first that all these edges are distinct. If $e=e^{\prime}$, we must have $x=x^{\prime}$, and thus $f=f^{\prime}$, and we have our conclusion. If $e_{y}^{\prime}=e$, we remove $x^{\prime}, y$ and obtain 42-24-20. If $e_{y}=e_{y}^{\prime}$, we also remove $x^{\prime}, y$ with the same result. So all these edges are distinct. If $e_{y}$ is not overlapping, removing $x, y^{\prime}$ gives 42-24-15-3. So both $e_{y}, e_{y}^{\prime}$ are overlapping. So $e_{y}$ intersects some edge $h$ on two vertices $u, v$ with external edges $e_{u}, e_{v}$. If all the edges defined so far are distinct, we remove $u, x, y^{\prime}$ to get 63-36-25-2,5. If the edge $e_{u}$ was already defined, this was $e_{x}, e^{\prime}, f^{\prime}$ or $e_{y}^{\prime}$, thus removing $v, x, y^{\prime}$ and deleting all the edges leave $x^{\prime}, y, u$ isolated, which gives 63-36-30, unless another edge was already defined. If this was $h$, removing $x, y^{\prime}$ would leave $e_{y}$ at least 2-degenerated, with a sum of 42-24-15-3. So $e_{v}$ was already defined. If $e_{u}=e_{y}^{\prime}$, we remove $x^{\prime}, y$, and thus $h, e_{u}$ become non plain (since $e_{v}$ is deleted), so 42-24-15-5. So, $e_{y}^{\prime}$ is distinct from $e_{u}, e_{v}$. Removing $x^{\prime}, y$ gives $42-24-15-5$, since $h, e_{y}^{\prime}$ become non plain. So, only the edge $h$ was already defined, and this was $e_{y}^{\prime}$ otherwise we remove $x^{\prime}, y$ to get $42-24-15-5$. But in this case, we remove $y, y^{\prime}$, in order to get 42-24-10-8 (since $e_{u}, e_{v}$ become at least special, and $e_{x}$ at least 2-degenerated.) $\square$ 
Claim 13 There is no vertex of degree two.

Proof. Let $x$ be a vertex of degree 2. It is contained in a non plain, and thus 1-degenerated, edge $e$ and in another edge $e_{x}$. If $e$ contains one vertex $y$ with degree three (incident to two other plain edges, by Claim 3), the edge $e_{x}$ is not plain, otherwise we remove $y$ to obtain 21-9-10-2,5. Thus $e_{x}$ is 1-degenerated. If removing $y$ leaves $e_{x} 2$ or 3-degenerated, we are done. So $e_{x}$ becomes 4-degenerated. Since $e_{x}$ is not special, this means that every edge incident to $y$ intersect $e_{x}$ on one vertex - but in this case we remove $x$, and the two other edges of $y$ become 1-degenerated, in all 21-2-15-6. Thus $e$ contains a vertex of degree 1 and three vertices of degree 2 .

- If there are plain and degenerated edges intersecting $e$, we pick a vertex $z$ of $e$ which is in two 1-degenerated edges. Removing $z$ creates a degenerated edge, and either creates another 1-degenerated or special edge, or creates a 2-degenerated edge. The sum is at least 21-2-15-2,5, with an additional -2 or $-2,5$ depending if the third edge incident with $e$ is plain or 1-degenerated.

- If all the edges intersecting $e$ are 1-degenerated edges, observe that this property spread over all the vertices of the connected component $C$ of $x$ in the hypergraph $H$. That is, every edge of $C$ is 1degenerated and the degree of every vertex of $C$ is at most two. Suppose now that there are $a$ vertices of degree 2 , then $C$ contains $2 a / 3$ edges, and thus $2 a / 3$ vertices of degree 1 . Only considering the vertices of degree 2 (and shrinking the edges), we obtain a 3 -uniform hypergraph with $a$ vertices and $2 a / 3$ edges. Thus, by Theorem 1 , there exists a transversal $T$ with $5 a / 12$ vertices. The gain is $105 a / 12-25 a / 3-2 a / 3$, in all $-a / 4$.

- If all the vertices of $e$ have degree 2, and all the edges intersecting $e$ are plain. Let $x, y, z$ be the vertices of $e$ with degree 2 , and $e_{x}, e_{y}, e_{z}$ their other (distinct) incident plain edges. If $e_{x}$ and $e_{y}$ do not intersect another edge on two vertices, removing $z$ gives 21-5-10-6. If $e_{x}$ intersects another edge $f$ on two vertices $u, v$. By Claim $8, u, v$ cannot be in all the edges $e_{x}, e_{y}, e_{z}$, so we assume that $u \notin e_{y}$. By Claim 11, removing $u$ gives 21-12-5-2,5 and makes $e$ at least 2-degenerated, but not 4-degenerated since $u \notin e_{y}$, so $-0,5$.

Claim 14 An edge is not both external and overlapping.

Proof. We suppose that all the degrees are 3 or 1 , and two edges $e$ and $f$ intersect on two vertices $x, y$ incident with other edges $e_{x}, e_{y}$. Moreover, $e_{x}$ intersect an edge $g$ on two vertices $u, v$. with additional edges $e_{u}$ and $e_{v}$. Let us prove first that all the edges defined so far are distinct. Clearly $e, f, g$ are distinct. By Claim 12, the only case when two edges could be the same is $g=e_{y}$. Let us call $z$ the vertex of $g$ distinct from $y, u, v$. Removing $z, x$ gives 42-24-15-5 since it leaves $e_{u}$ and $e_{v}$ degenerated.

All these edges are now distinct, and consequently, if $e_{v}$ does not intersect an edge on two vertices, we conclude by removing $u, y$ with a sum of 42-24-15-3. So $e_{v}$ intersects another edge $h$ on two vertices $a, b$, each of them with external edges $e_{a}, e_{b}$.

Observe that if all these edges are distinct, removing $a, u, y$ will give 63-36-25-2,5.

So, some of these edges are the same. If $h$ was already defined, removing $v, y$ gives 42-24-15-5 since $e_{a}$ and $e_{b}$ become degenerated, or, if $e_{a}$ or $e_{b}$ were defined, $a$ or $b$ becomes isolated. The only case left, by Claim 12, could be $e_{a}=e$. But, we remove $y, v$ to obtain 42-24-15-5 since $h, e_{u}$ become degenerated.

Claim 15 There are no overlapping edges.

Proof. Let two edges $e$ and $f$ intersecting on two vertices $x, y$ incident with other edges $e_{x}, e_{y}$. Let $z$ be a vertex of $e_{x}$ which is not a vertex of $e, f, e_{y}$. If none of the three edges incident to $z$ is overlapping, we split $z$ into three vertices of degree 1 , which gives $+10-9$, and remove $x$, which is 21-9-10-3. So let $e_{z}, f_{z}$ be the other edges incident to $z$. Note that $e_{z} \cap f_{z}=\{z\}$ by Claim 12. Suppose that $e_{z}$ intersect an edge $g$ on two vertices $u, v$ incident with other edges $e_{u}, e_{v}$. If all these edges are distinct, we remove $u, x$, with -24 (edges) -10 (vertices) and -8,5 ( $e_{y}, e_{v}$ become 1 -degenerated and $f_{z}$ becomes at least special). Thus, some of these edges must be the same. By Claim 12 and Claim 14, the only case is $g=e$, and we can conclude by removing $z, y$ since we get $42-24-15-6$. 
Claim 16 All the edges are plain.

Proof. Let $e$ be a non plain, and thus 1-degenerated edge. The other vertices have degree 3 and must be incident to other plain edges. Let $x, y, z$ be the vertices of $e$ with degree 3 , and $e_{x}, f_{x}, e_{y}, f_{y}, e_{z}, f_{z}$, be the edges incident to $x, y, z$ different from $e$. They are all distinct since $e$ is not special. Since $e_{x}, f_{x}, e_{y}, f_{y}$ do not intersect another edge on two vertices, we split $x$ and $y(+8)$ and remove $z$ which gives 21-9-20.

Finally, $H$ is a 3 -uniform and 3-regular hypergraph with no overlapping edges. We now obtain a contradiction as follows. Let $x \in V(H)$ be arbitrary. Let $H_{x}$ be a hypergraph with vertex set $N(x)$ (i.e. all vertices which lie in an edge containing $x$, but $x \notin N(x)$ ), and edge set $\{V(e) \cap N(x):|V(e) \cap N(x)| \geq 2\}$ (i.e. all edges which contain at least 2 vertices from $N(x)$, are restricted to $N(x)$ ).

All edges in $H_{x}$ have size 2, because of the following. Clearly every edge has size at least 2, so assume, for the sake of contradiction, that $e$ is an edge in $H_{x}$ of size at least 3 . By Claim 15, we see that $|V(e)|=3$ in $H_{x}$. Let $e^{\prime}$ be the edge in $H$, with the property that $V(e) \subset V\left(e^{\prime}\right)$, and let $w$ be the unique vertex in $V\left(e^{\prime}\right)-N(x)$. Now we delete $x, w$, which gives 42-24-10-9, since the remaining vertices of $e$ now have degree one and thus their incident edges become degenerated.

So we can think of $H_{x}$ as a graph. Now $H_{x}$ is a graph with 9 vertices and 3 vertex disjoint cycles of length 3 , because of the following. By Claim 15 , we get that $V\left(H_{x}\right)=9$. If there was an independent set $X$ in $H_{x}$ of size at least 4 , we would delete $x$, which gives 21-12-5 and split the vertices of $X$, each one for a -1 . So $H_{x}$ is a graph on 9 vertices with stability at most 3 and maximum degree 2 , only three disjoint 3-circuits can achieve this.

Now let $e \in E(H)$ be an edge such that $|V(e) \cap N(x)|=2$ and let $y \in V(e)-N(x)$ be arbitrary. Let $w_{1} \in V(H)$ belong to a 3-cycle in $H_{x}$, which does not contain the edge $(V(e) \cap N(x))$, and let $w_{2} \in V(H)$ belong to a 3 -cycle in $H_{x}$, which does not contain the edge $(V(e) \cap N(x))$ or the vertex $w_{1}$. Now, we delete $x, y$, for a sum of 42-24-10-6, since the vertices in $V(e) \cap N(x)$ now have degree one, and in particular their incident edges become degenerated. To achieve the bound, split the vertices $w_{1}$ and $w_{2}$, each for a $-1$.

Another way of proving the $21|T| \leq 5 n+4 m$ formula is to allow edges of size 3 and 2 . Let $H_{1}$ be the hypergraph, with vertices $\left\{x_{1}, x_{2}, x_{3}, x_{4}, x_{5}\right\}$ and edges $\left\{\left\{x_{1}, x_{2}, x_{3}\right\},\left\{x_{1}, x_{4}, x_{5}\right\},\left\{x_{2}, x_{3}, x_{4}, x_{5}\right\}\right\}$, and let $H_{2}$ be the hypergraph with vertices $\left\{x_{1}, x_{2}\right\}$ and one edge $\left\{x_{1}, x_{2}\right\}$. For a hypergraph, $H$, where all edges have size at least 2 and at most 4 , let $e_{i}(H)$ denote the number of edges in $H$ of size $i(i=2,3,4)$. Let $h_{1}(H)$ be the number of components of $H$, which are isomorphic to $H_{1}$, and let $h_{2}(H)$ be the number of components of $H$, which are isomorphic to $H_{2}$.

Theorem 4 There exists a transversal, $T$ of $H$ such that

$$
21|T| \leq 5|V(H)|+10 e_{2}(H)+6 e_{3}(H)+4 e_{4}(H)+h_{1}(H)+h_{2}(H)
$$

The proof of the above theorem is however quite technical and of equal length to the proof Theorem 3 , so we omit it from this paper.

\section{$3 \quad$ Asymptotics}

Our goal in this section is to bound the value of $c_{k}$. The next upper bound can also be derived from a result of Johnson and Lovász: $\tau(H) \leq(1+\ln (d)) \tau^{*}(H)$ where $H$ is a hypergraph, $\tau(H), \tau^{*}(H)$ are respectively the transversality and the fractional transversality of $H$ and $d$ is the maximum degree of $H$, see $[8]$.

Theorem $5 \quad c_{k} \leq \frac{1+\ln (k)}{k}$ 
Proof. Let $H$ be a $k$-uniform hypergraph, with $n$ edges and $n$ vertices. Define $S(G)=\sum_{x \in V(G)} d(x)$, for all hypergraphs $G$, and note that $S(H)=k n$. Let $H^{\prime}$ be a $k$-uniform hypergraph with $S\left(H^{\prime}\right) \leq i n$, where $i$ is an integer. We will now show that there exists a set $X$, such that $|X| \leq \frac{n}{i k}$ and $S\left(H^{\prime} \backslash X\right) \leq(i-1) n$. If $S\left(H^{\prime}\right)>(i-1) n$, then there exists a vertex $x$ in $H^{\prime}$, such that $d(x) \geq i$. Now deleting this vertex (and edges incident with it) makes the sum $S$ drop by $d(x) k \geq i k$. So by keep deleting such vertices until the sum is at most $(i-1) n$, we delete at most $\frac{n}{i k}$ vertices.

By the above we note that there exists a transversal of size at most the following.

$$
\begin{aligned}
\sum_{i=1}^{k} \frac{n}{k i} & =\frac{n}{k} \sum_{i=1}^{k} \frac{1}{i} \\
& \leq \frac{n}{k}(1+\ln (k)) \\
& =n \frac{1+\ln (k)}{k}
\end{aligned}
$$

This completes the proof.

Theorem 6 For any $\varepsilon>0, c_{k}>\frac{(1-\varepsilon) \ln (k)}{k}$ for sufficiently large $k$.

Proof. Let $1>\varepsilon>0$ be arbitrary. We will now show that $c_{k}>\frac{(1-\varepsilon) \ln (k)}{k}$, for all sufficiently large $k$. If $e$ is the base of the natural logarithm, then given $\varepsilon$, there exists a constant $A_{\varepsilon}$, such that $\left(1-\alpha^{-1}\right)^{\alpha}>(1-\varepsilon) / e$, when $\alpha \geq A_{\varepsilon}$. Furthermore $\left(1-\alpha^{-1}\right)^{\alpha}<1 / e$ is true for all $\alpha \geq 1$. We will often use the above, without explicitly stating it.

By the above we let $a$ be some number such that the following holds.

$$
\begin{aligned}
& e^{-1} e^{-(\varepsilon / 2) /(1-\varepsilon)}<\left(1-a^{-1}\right)^{a} \\
& \Downarrow \\
& \Downarrow \\
& \frac{(\varepsilon / 2)-1}{1-\varepsilon}<\operatorname{aln}\left(\frac{a-1}{a}\right) \\
& \Downarrow \\
& (\varepsilon / 2-1) \ln (k)<(1-\varepsilon) \ln (k) \operatorname{aln}\left(\frac{a-1}{a}\right) \\
& 1-k^{-(1-\varepsilon / 2)}>1-e^{\left[(1-\varepsilon) \ln (k) \operatorname{aln}\left(\frac{a-1}{a}\right)\right]}
\end{aligned}
$$

Given $a$ we let $k$ be large enough such that the following holds.

$$
(1-\varepsilon)(\ln (k))(\ln (a k))-k^{\varepsilon / 2}<0
$$

This can clearly be done as $k^{\varepsilon / 2}$ grows faster than any poly-log function. We will now show that a random hypergraph on $n=a k$ vertices and with $n$ edges, all of size $k$, has probability strictly less than 1 , of having a transversal of size $z=n \frac{(1-\varepsilon) \ln (k)}{k}$. So there must exist $k$-uniform hypergraphs on $n=a k$ vertices and with $n$ edges, which do not have any transversal of size $z$. This would prove the theorem.

Let $H$ be a hypergraph, with $n=a k$ vertices and with $n$ edges, all chosen at random (an edge may be chosen several times). Let $H_{1}, H_{2}, \ldots, H_{n}$ denote the edges. Let $Z$ be $z$ vertices chosen at random (a vertex may be picked several times). There are $n^{z}$ possible ways of choosing $Z$. The probability that $Z \cap H_{i}=\emptyset$ is clearly $[(n-k) / n]^{z}$, as each vertex in $Z$ has probability $(n-k) / n$ of not lying in $H_{i}$. So the probability that $Z \cap H_{i} \neq \emptyset$ for all $i=1,2, \ldots, n$, is the following. Since the $H_{i}$ are chosen independently, we have:

$$
P\left(Z \cap H_{i} \neq \emptyset, i=1,2, \ldots, n\right)=\left(1-\left(\frac{n-k}{n}\right)^{z}\right)^{n}
$$

Let $\mathcal{Z}$ contain all possible $Z$ 's (i.e. $|\mathcal{Z}|=n^{z}$, and $Z \in \mathcal{Z}$ if and only if $Z$ contains exactly $z$ elements, some of which may be identical). As $Z \in \mathcal{Z}$ was chosen with equal probability, namely $1 / n^{z}$, we get the following. 


$$
\begin{aligned}
& \frac{1}{n^{z}} \sum_{Z \in \mathcal{Z}} P\left(Z \cap H_{i} \neq \emptyset, i=1,2, \ldots, n\right)=\left(1-\left(\frac{n-k}{n}\right)^{z}\right)^{n} \\
& \Downarrow \\
& \sum_{Z \in \mathcal{Z}} P\left(Z \cap H_{i} \neq \emptyset, i=1,2, \ldots, n\right)=(a k)^{z}\left(1-\left(\frac{a-1}{a}\right)^{z}\right)^{a k} \\
& \Downarrow \\
& P(H \text { has a transversal of size at most } z) \leq(a k)^{z}\left(1-\left(\frac{a-1}{a}\right)^{z}\right)^{a k}
\end{aligned}
$$

Let $\gamma=(a k)^{z}\left(1-\left(\frac{a-1}{a}\right)^{z}\right)^{a k}$, and recall that $z=a(1-\varepsilon) \ln (k)$, which implies the following (by $(A)$ and $(B))$ :

$$
\begin{aligned}
\ln (\gamma) & =a(1-\varepsilon) \ln (k) \ln (a k)+a k \ln \left(1-\left(\frac{a-1}{a}\right)^{a(1-\varepsilon) \ln (k)}\right) \\
& =a(1-\varepsilon) \ln (k) \ln (a k)+a k \ln \left(1-e^{a(1-\varepsilon) \ln (k) \ln \left(\frac{a-1}{a}\right)}\right) \\
& <a(1-\varepsilon) \ln (k) \ln (a k)+a k \ln \left(1-k^{-(1-\varepsilon / 2)}\right) \\
& =a(1-\varepsilon) \ln (k) \ln (a k)+\frac{a k}{k^{(1-\varepsilon / 2)}} \ln \left(\left(1-k^{-(1-\varepsilon / 2)}\right)^{k^{(1-\varepsilon / 2)}}\right) \\
& <a(1-\varepsilon) \ln (k) \ln (a k)+a k^{1-1+\varepsilon / 2} \ln \left(e^{-1}\right) \\
& =a(1-\varepsilon) \ln (k) \ln (a k)-a k^{\varepsilon / 2} \\
& <0
\end{aligned}
$$

Therefore the probability that $H$ contains a transversal is strictly less than 1 , and the proof is complete.

Note that in the above proof if $\varepsilon=0.6$, then $c_{k} \geq \frac{0.4 \times \ln (k)}{k}$, when $a=2.8$ and $k \geq 4800000$ (simply insert these values in the proof). It is not difficult to check that $\gamma<1$ for all smaller values of $k$ in the proof as well (using a computer). Therefore the following is true.

Corollary $3 \quad c_{k}>\frac{0.4 \times \ln (k)}{k}$, for all $k \geq 2$.

Furthermore the previous results imply the following.

Corollary $4 \lim _{k \rightarrow \infty} \frac{c_{k}}{\ln (k) / k}=1$.

\section{$4 \quad$ A general formula?}

Let $k>1$ be a fixed integer. The set $A_{k}$ of pairs $a, b$ such that every $k$-uniform hypergraphs with $n$ vertices and $m$ edges has a transversal with at most $a n+b m$ vertices has been studied by Chvátal and McDiarmid in [5]. This closed convex set has infinitely many extreme points. They described the two last (with largest $b$ ) extreme points of $A_{k}$, for all $k$. They also prove that the three last points $(a, b)$ of $A_{3}$ are $(0,1),(1 / 4,1 / 4),(1 / 3,1 / 6)$. Our proof gives in fact that the three last points of $A_{4}$ are $(0,1),(1 / 6,1 / 3),(5 / 21,4 / 21)$. The two first points are from [5]. The last one belongs to $A_{4}$ as we have seen. We just have to check that it is extreme. To see that, observe that the hypergraph on $\{1,2,3,4,5,6\}$, with edge set $\{1234,3456,5612\}$ is sharp for $(1 / 6,1 / 3)$ and $(5 / 21,4 / 21)$. Moreover, the complement of the Fano plane is not sharp for $(1 / 6,1 / 3)$ and sharp for $(5 / 21,4 / 21)$. Thus, $(5 / 21,4 / 21)$ is an extreme point of $A_{4}$. In [3] was proved that $(2 / 9,2 / 9) \in A_{4}$. This can be easily derived from our bound since $(2 / 9,2 / 9)=2 / 9(1 / 6,1 / 3)+7 / 9(5 / 21,4 / 21)$. The full description of $A_{k}$, for $k \geq 3$, seems a very hard problem. However, Chvátal and McDiarmid conjecture that for every $\varepsilon>0, A_{k}$ has finitely many elements for which $a>\varepsilon$.

We would like to concentrate on the following question: Is there an element (or more) of $A_{k}$ which gives the constant $c_{k}$ ? Precisely, is it always possible to find $(a, b) \in A_{k}$ for which $a+b=c_{k}$ ? As we have 
calculated so far, there is such a pair in $A_{2}, A_{3}$ and $A_{4}$. But we do not see any reason why this should be the case for larger values of $k$.

Finally, we will make a guess concerning the value of $c_{5}$. Consider the non zero quadratic residues modulo 11 , that is $Q=\{1,3,4,5,9\}$. And form the hypergraph on $\{0, \ldots, 10\}$ which edges are the sets $Q+i$, where $i=0 . .10$. It is not hard to check, by enumeration, that every subset on three vertices avoid one edge, and consequently, that the minimum transversal of this hypergraph has 4 vertices. Thus $4 / 11 \leq c_{5}$, and we conjecture that equality holds.

Acknowledgement. We thank Odile Favaron for introducing us the problem. This collaboration started during the graph conference held in Odense, organised by Joergen Bang-Jensen and Bjarne Toft.

\section{References}

[1] N. Alon, Transversal numbers of uniform hypergraphs, Graphs Combin., 6 (1990), 1-4.

[2] D. Archdeacon, J. Ellis-Monaghan, D. Fisher, D. Froncek, P.C.B. Lam, S. Seager, B. Wei, R. Yuster, Some remarks on domination, J. Graph Theory, 46 (2004), 207-210.

[3] G.J. Chang and F.C. Lai, An upper bound for the transversal numbers of 4-uniform hypergraphs, J. Combin. Theory Ser. B, 50 (1990), 129-133.

[4] P.C.B. Lam and B. Wei, On the total domination number of graphs, preprint.

[5] V. Chvátal and C. McDiarmid, Small transversals in hypergraphs, Combinatorica, 12 (1992), $19-26$.

[6] O. Favaron, M. Henning, C. Mynhart and J. Puech, Total domination in graphs with minimum degree three, J. Graph Theory, 34 (2000), 9-19.

[7] D. Fisher, Fractional dominations and fractional total dominations of graph complements, Discrete Appl. Math., 122 (2002), 283-291.

[8] A. Schrijver, Combinatorial optimization. Polyhedra and efficiency, Springer-Verlag, Berlin, 2003.

[9] P.D. Seymour, On the two-colouring of hypergraphs, Quart. J. Math. Oxford Ser. (2), 25 (1974), 303-312. 